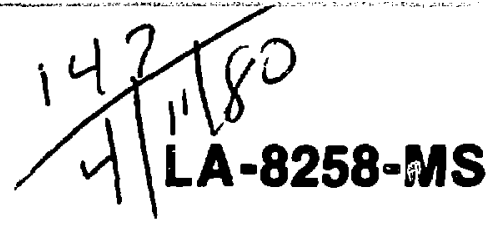

Informal Report

\section{DR. 1037}

\title{
A Novel Approach to the Computation of Axisymmetric Countercurrent Patterns in Gas Centrifuges
}

\section{MASTER}

先

$\frac{2}{\omega}$

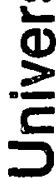




\title{
A NOVEL APPROACH TO THE COMPUTATION OF AXISYMMETRIC COUNTERCURRENT PATTERNS IN GAS CENTRIFUGES
}

\author{
by \\ L. D. Cloutman
}

\begin{abstract}
ABSTRAC, T
We present a numerical algorithm based on $f i-$ nite Fourier-Chebyshev transforms for solving the total reflux, thermal-drive ceritrifuge problem. The method should be capable of providing steadystate solutions with excellent resolution in a few minutes of computer time. The same technique may be easily applied to other problems, including the isotope diffusion equation. A simple diffusion equation is solved to show certain aspects of the numerical method, in particular, its excellent convergence properties.
\end{abstract}

\section{INTRODUCTION}

We present a method for numerically solving a system of partial differential equations that is applicable to a wide variety of problems, including some nonlinear effects. The particular application that we use to present the method is the steady-stace flow profile inside a gas centrifuge. The method directly solves for the steady-state solution, and therefore should be computationally more efficient than the technique of allowing a time-dependent finite difference prugram seek the steady state through a transient.

The centrifuge countercurrent problem has two features that make it difficult to treat numericaliy. First, the density can vary by a large factor across the machine, depending on the operating conditions. This means that special attention must be given to the diffusion terms to obtain stable difference methods. Second, the existence of thin boundary layers on the rotor wall and endcaps requires the use of a variable mesh to obtain good accurecy. Here we show how both problems are easily handled by the finite Fourier tiansform technique. 
The basic idea in the finite Fourier transform method is to reduce the two-dimensional partial differential equations to an infinite set of ordinary differential equations. The solutions of the partial differential equations are expanded as a series of some suitable orthogonal functions, where the argument of the orthogonal function is one independent variable and the expansion coefficients are functions of the secord variable. Substitution of the series into the partial differential equations, followed by integration over the first variable, produces an infinite set of ordinary differential equations in the second variable, whose solutions are the expansion coefficients. The solution is approximated by a truncated series, leaving a finite set of ordinary differential equations, which are solved numerically.

In Sec. II we derive the set of ordinary differential equations for tive centrifuge problem and specify boundary conditions. In Sec. III we describe the proposed numerical scheme for solving the ordinary differential equations. A simple problein is solved and discussed in Sec. IV to show some of the properties of the method.

\section{THE FLOW EQUATIONS}

Assume that the countercurrent in a gas centrifuge is adequately described by the equations of continuum fluid dynamics. The full nonlinear equations in cylindrical coordinates are

$$
\begin{aligned}
\frac{\partial \rho}{\partial r}+\frac{1}{r} \frac{\partial}{\partial r}(r u \rho)+\frac{\partial}{\partial z}(w \rho)= & 0 \\
\rho \frac{\partial u}{\partial t}+\rho\left(u \frac{\partial u}{\partial r}+w \frac{\partial u}{\partial z}-\frac{v^{2}}{r}\right)= & -\frac{\partial p}{\partial r}+2 \mu\left[\frac{\partial^{2} u}{\partial r^{2}}+\frac{1}{r}\left(\frac{\partial u}{\partial r}-\frac{u}{r}\right)\right]+\mu \frac{\partial}{\partial z}\left(\frac{\partial u}{\partial z}+\frac{\partial w}{\partial r}\right) \\
& +\lambda \frac{\partial}{\partial r}\left(\frac{1}{r} \frac{\partial r u}{\partial r}+\frac{\partial w}{\partial z}\right) \\
\rho \frac{\partial v}{\partial t}+\rho\left(u \frac{\partial v}{\partial r}+w \frac{\partial v}{\partial z}+\frac{u v}{r}\right)= & \mu\left[\frac{\partial^{2} v}{\partial z^{2}}+\frac{\partial^{2} v}{\partial r^{2}}-\frac{\partial}{\partial r}\left(\frac{v}{r}\right)+\frac{2}{r}\left(\frac{\partial v}{\partial r}-\frac{v}{r}\right)\right]
\end{aligned}
$$




$$
\begin{aligned}
\rho \frac{\partial w}{\partial t}+\rho\left(u \frac{\partial w}{\partial r}+w \frac{\partial w}{\partial z}\right)= & -\frac{\partial p}{\partial z}+\mu\left[2 \frac{\partial^{2} w}{\partial z^{2}}+\frac{1}{r} \frac{\partial}{\partial r}\left(r \frac{\partial u}{\partial z}\right)+\frac{1}{r} \frac{\partial}{\partial r}\left(r \frac{\partial w}{\partial r}\right)\right] \\
& +\lambda \frac{\partial}{\partial z}\left(\frac{1}{r} \frac{\partial r u}{\partial r}+\frac{\partial w}{\partial z}\right),
\end{aligned}
$$

and

$$
\begin{aligned}
\rho \frac{\partial I}{\partial t} & +\rho\left(u \frac{\partial I}{\partial r}+w \frac{\partial I}{\partial z}\right)=-p\left(\frac{1}{r} \frac{\partial(r u)}{\partial r}+\frac{\partial w}{\partial z}\right)+\frac{\tau}{c_{v} r} \frac{\partial}{\partial r} r \frac{\partial I}{\partial r}+\frac{\tau}{c_{v}} \frac{\partial^{2} I}{\partial z^{2}} \\
& +\lambda\left(\frac{1}{r} \frac{\partial(r u)}{\partial r}+\frac{\partial w}{\partial z}\right)^{2}+\mu\left[2\left(\frac{\partial u}{\partial r}\right)^{2}+2\left(\frac{\partial w}{\partial z}\right)^{2}+2\left(\frac{u}{r}\right)^{2}+\left(\frac{\partial v}{\partial z}\right)^{2}+\left(\frac{\partial u}{\partial z}\right)^{2}\right. \\
& \left.+\left(\frac{\partial w}{\partial r}\right)^{2}+2 \frac{\partial u}{\partial z} \frac{\partial w}{\partial r}+\left(\frac{\partial v}{\partial r}\right)^{2}-\frac{2 v}{r} \frac{\partial v}{\partial r}+\left(\frac{v}{r}\right)^{2}\right]
\end{aligned}
$$

where $\rho, u, v, w, I$ and $p$ are respectively the gas density, the radial, tangential, and axial velocity components, the specific internal energy and the pressure. We have assumed axial symmetry although all three velocity components are retained. The first and second viscosity coefficients, the coefficient of viscosity, the second coefficient of viscosity, and the thermal conductivity, $\mu, \lambda$, and $\tau$ respectively, are assumed to be constant. The ideal gas equation of state,

$$
p=(\gamma-1) \rho I,
$$

is used in our calculations.

We will linearize the flow equations by breaking the solution into a zeroth order part and a perturbation term, and then keep only those terms linear in the perturbation quantities. For the zeroth order solution, denoted by the subscript 0 , we assume rigid body rotation and hydiostatic equilibrium in an isothermal fluid. Then

$$
\frac{\partial p_{0}}{\partial r}=p_{0} \omega^{2} r
$$


where $\omega$ is the angular velocity of the centrifuge. This may be integrated to give

$$
\begin{aligned}
P_{0} & =P_{\text {wall }} e^{-A^{2}\left(1-\frac{r^{2}}{a^{2}}\right)} \\
& \equiv p_{\text {wall }} n,
\end{aligned}
$$

where

$$
A^{2}=\frac{a^{2} \omega^{2}}{2(\gamma-1) I_{0}} \equiv U^{2} / 2
$$

Here, $\gamma$ is the ratio of specific heats, and $a$ is the centrifuge rotor radius. From the equation of state and the isothermal assumption,

$$
\therefore=\rho_{\text {wall }} n \cdot
$$

The complete solution may be written

$$
\begin{aligned}
& \vec{u}=\vec{u}_{0}+\vec{u}^{\prime}=(0, w r, 0)+\left(u^{\prime}, v^{\prime}, w^{\prime}\right), \\
& I=I_{0}+I^{\prime}, \\
& p=p_{0}+p^{\prime}=p_{w a l} n+p^{\prime},
\end{aligned}
$$

and

$$
\rho=\rho_{0}+\rho^{\prime}=\rho_{\text {wall }}{ }^{n+\rho^{\prime},}
$$

where primes denote perturbations from the zeroth order solution, assumed to be small. We will use the linearized equation of state

$$
\rho^{\prime}=\frac{p^{\prime}}{(\gamma-1) I_{0}}-\frac{I^{\prime} \rho_{0}}{I_{0}}
$$

to eliminate $\rho^{\prime}$. 
We will linearize Eq. (1)-(5) by dropping all terms quadratic in primed quantities. Upon linearization of Eq. (1), the equation of continuity, we find that

$$
\frac{\partial \rho^{\prime}}{\partial t}+\frac{1}{r} \frac{\partial}{\partial r}\left(r \rho_{0} u^{\prime}\right)+\frac{\partial}{\partial z}\left(\rho_{0} w^{\prime}\right)+0
$$

Equation (2) becomes

$$
\begin{aligned}
\rho_{0} \frac{\partial u^{\prime}}{\partial t} & -\rho^{\prime} \omega^{2} r-2 \rho_{0} v^{\prime} \omega=-\frac{\partial p^{\prime}}{\partial r}+\mu\left[\frac{2}{r} \frac{\partial}{\partial r}\left(r \frac{\partial u^{\prime}}{\partial r}\right)-\frac{2 u^{\prime}}{r^{2}}+\frac{\partial^{2} u^{\prime}}{\partial z^{2}}+\frac{\partial^{2} w^{\prime}}{\partial r \partial z}\right. \\
& \left.+\lambda\left(\frac{\partial}{\partial r} \frac{1}{r} \frac{\partial}{\partial r}\left(r u^{\prime}\right)+\frac{\partial^{2} w^{\prime}}{\partial r \partial z}\right)\right] .
\end{aligned}
$$

Next we linearize Eq.(3), obtaining

$$
\rho_{0} \frac{\partial v^{\prime}}{\partial t}+2 \rho_{0} \omega u^{\prime}=\mu\left[\frac{\partial^{2} v^{\prime}}{\partial z^{2}}+\frac{\partial^{2} v^{\prime}}{\partial r^{2}}+\frac{1}{r} \frac{\partial v^{\prime}}{\partial r}-\frac{v^{\prime}}{r^{2}}\right] \text {. }
$$

The linearized axial momentum equation is

$$
\begin{aligned}
\rho_{0} \frac{\partial w^{\prime}}{\partial t}= & -\frac{\partial p^{\prime}}{\partial z}+\mu\left[2 \frac{\partial^{2} W^{\prime}}{\partial z^{2}}+\frac{1}{r} \frac{\partial}{\partial r}\left(r \frac{\partial u^{\prime}}{\partial z}\right)+\frac{1}{r} \frac{\partial}{\partial r} r \frac{\partial w^{\prime}}{\partial r}\right] \\
& +\lambda \frac{\partial}{\partial z}\left(\frac{1}{r} \frac{\partial r u^{\prime}}{\partial r}+\frac{\partial w^{\prime}}{\partial z}\right) .
\end{aligned}
$$

The viscous terms can be simplified by assuming

$$
\nabla \cdot\left(\rho_{0} \vec{u}^{\prime}\right)=0
$$

This condition is exact for the steady-state linear solution, and experience shows it is a good approximation in the transient phases. Equation (20) implies

$$
\nabla \cdot \vec{u}^{\prime}=\frac{-u^{2} r u^{\prime}}{a^{2}} .
$$


From (21),

$$
\frac{\partial^{2} w^{\prime}}{\partial r \partial z}=-\frac{\partial}{\partial r}\left[\frac{1}{r} \frac{\partial}{\partial r}\left(r u^{\prime}\right)+\frac{u^{2} r u^{\prime}}{a^{2}}\right]
$$

and

$$
\frac{1}{r} \frac{\partial}{\partial r}\left(r \frac{\partial u^{\prime}}{\partial z}\right)=-\frac{\partial}{\partial z}\left[\frac{\partial w^{\prime}}{\partial z}+\frac{u^{2} r u^{\prime}}{a^{2}}\right] \text {. }
$$

Upon substituting Eq. (21) and Eq. (22) into Eq. (17), we find that

$$
\begin{aligned}
\rho_{0} \frac{\partial u^{\prime}}{\partial t}= & -\frac{\partial p^{\prime}}{\partial r}+\rho^{\prime} \omega^{2} r+2 \rho_{0} \omega v^{\prime}-\frac{\lambda u^{2}}{a^{2}} \frac{\partial}{\partial r}\left(r u^{\prime}\right)+\mu\left[\frac{1}{r} \frac{\partial}{\partial r} r \frac{\partial u^{\prime}}{\partial r}+\frac{\partial^{2} u^{\prime}}{\partial z^{2}}\right. \\
& \left.-\frac{u^{\prime}}{r^{2}}-\frac{U^{2}}{a^{2}} \frac{\partial}{\partial r}\left(r u^{\prime}\right)\right] .
\end{aligned}
$$

Equations (21) and (23) may be substituted into Eq. (19) to obtain

$$
\rho_{0} \frac{\partial w^{\prime}}{\partial t}=-\frac{\partial p^{\prime}}{\partial z}+\mu\left[\frac{\partial^{2} w^{\prime}}{\partial z^{2}}+\frac{1}{r} \frac{\partial}{\partial r}\left(r \frac{\partial w^{\prime}}{\partial r}\right)-\frac{U^{2} r}{a^{2}} \frac{\partial u^{\prime}}{\partial z}\right]-\frac{\lambda U^{2} r}{a^{2}} \frac{\partial u^{\prime}}{\partial z}
$$

Linearizing Eq. (5), we obtain the linearized energy equation,

$$
\rho_{0} \frac{\partial I^{\prime}}{\partial t}=-p_{0}\left(\frac{1}{r} \frac{\partial}{\partial r}\left(r u^{\prime}\right)+\frac{\partial W^{\prime}}{\partial z}\right)+\frac{\tau}{c_{v}}\left[\frac{1}{r} \frac{\partial}{\partial r}\left(r \frac{\partial I^{\prime}}{\partial r}\right)+\frac{\partial^{2} I^{\prime}}{\partial z^{2}}\right] .
$$

We will transform our equations to dimensionless form using the following definitions :

$$
\begin{aligned}
& u^{\prime}=\omega a \delta u, \\
& v^{\prime}=\omega a \delta u,
\end{aligned}
$$




$$
\begin{aligned}
& w^{\prime}=\omega a \delta w, \\
& z=a z^{\prime} \\
& r=a r^{\prime} \\
& T^{\prime}=T_{0} \delta \theta, \\
& p^{\prime}=p_{0} \delta p, \\
& \omega t=t^{\prime}, \\
& B=1+\lambda / \mu,
\end{aligned}
$$

and

$$
\operatorname{Re}=\rho_{\text {wal1 }} \omega \mathrm{a}^{2} / \mu .
$$

Transforming the energy equation (26) and using Eq. (21), we obtain

$$
\frac{\partial \delta \theta}{\partial t^{\prime}}=(\gamma-1) u^{2} r^{\prime} \delta u+\frac{\gamma}{\operatorname{Re} \operatorname{Pr} \eta}\left[\frac{1}{r^{\prime}} \frac{\partial}{\partial r^{\prime}}\left(r^{\prime} \frac{\partial \delta \theta}{\partial r^{\prime}}\right)+\frac{\partial^{2} \delta \theta}{\partial z^{\prime 2}}\right]
$$

where the Prandtl number $\operatorname{Pr}$ is defined by $\tau=c_{p} / \operatorname{Pr}$.

Next we will transform the continuity equation. Using Eq. (15) to eliminate $\rho^{\prime}$, we may rewrite the continuity equation, Eq. (16), as

$$
\frac{\partial \delta p}{\partial t^{\prime}}-\frac{\partial \delta \theta}{\partial t^{\prime}}+\frac{1}{r^{\prime} n} \frac{\partial}{\partial r^{\prime}}\left(r^{\prime} n \delta u\right)+\frac{\partial}{\partial z^{\prime}} \delta w=0 .
$$

The radial momentum equation, Eq. (24) becomes

$$
\begin{aligned}
\frac{\partial \delta u}{\partial t^{\prime}}= & -\frac{1}{u^{2}} \frac{\partial \delta p}{\partial r^{\prime}}-r^{\prime} \delta \theta+2 \delta v-\frac{B u^{2}}{\operatorname{Re} n} \frac{\partial}{\partial r^{\prime}} r^{\prime} \delta u+\frac{1}{\operatorname{Re} n}\left[\frac{1}{r^{\prime}} \frac{\partial}{\partial r^{\prime}}\left(r^{\prime} \frac{\partial \delta u}{\partial r^{\prime}}\right)\right. \\
& \left.+\frac{\partial^{2} \delta u}{\partial z^{\prime 2}}-\frac{\delta u}{r^{\prime 2}}\right] .
\end{aligned}
$$

The tangential momentum equation, Eq. (18), transforms to

$$
\frac{\partial \delta v}{\partial t^{\prime}}=-2 \delta u+\frac{1}{\operatorname{Re} n}\left[\frac{1}{r^{\prime}} \frac{\partial}{\partial r^{\prime}}\left(r^{\prime} \frac{\partial \delta v}{\partial r^{\prime}}\right)+\frac{\partial^{2} \delta v}{\partial z^{\prime 2}}-\frac{\delta v}{r^{\prime 2}}\right]
$$


Finally, the axial momentum equation (25) becomes

$$
\frac{\partial \delta w}{\partial t^{\prime}}=-\frac{1}{u^{2}} \frac{\partial \delta p}{\partial z^{\prime}}-\frac{B U^{2}}{\operatorname{Re} \eta} r^{\prime} \frac{\partial \delta u}{\partial z^{\prime}}+\frac{1}{\operatorname{Re} \eta}\left[\overline{r^{\prime}}\left(r^{\prime} \frac{\partial \delta w}{\partial r^{\prime}}\right)+\frac{\partial^{2} \delta w}{\partial z^{\prime 2}}\right] \text {. }
$$

In order to make the notation less cumbersome, we drop the $\delta$ 's and primes at this point. We also drop the time derivatives because we are interested only in steady-state solutions. The equations that we want to solve are

$$
\begin{aligned}
& \left(u^{2} r+\frac{1}{r}\right) u+\frac{\partial u}{\partial r}+\frac{\partial w}{\partial z}=0 \\
& (r-1) u^{2} r u+\frac{\gamma}{\operatorname{Re} \operatorname{Pr} \eta}\left[\frac{\partial^{2} \theta}{\partial z^{2}}+\frac{\partial^{2} \theta}{\partial r^{2}}+\frac{1}{r} \frac{\partial \theta}{\partial r}\right]=0 \\
& -2 u+\frac{1}{\operatorname{Re} \eta}\left[\frac{\partial^{2} v}{\partial z^{2}}+\frac{\partial^{2} v}{\partial r^{2}}+\frac{1}{r} \frac{\partial v}{\partial r}-\frac{v}{r^{2}}\right]=0 \\
& -\frac{1}{u^{2}} \frac{\partial p}{\partial r}-r \theta+2 v-\frac{B u^{2}}{\operatorname{Re} n}\left[r \frac{\partial u}{\partial r}+u\right]+\frac{1}{\operatorname{Re} \eta}\left[\frac{\partial^{2} u}{\partial z^{2}}+\frac{\partial^{2} u}{\partial r^{2}}+\frac{1}{r} \frac{\partial u}{\partial r}-\frac{u}{r^{2}}\right]=0,
\end{aligned}
$$

and

$$
-\frac{1}{U^{2}} \frac{\partial p}{\partial z}-\frac{B U^{2}}{\operatorname{Re} \eta} r \frac{\partial u}{\partial z}+\frac{1}{\operatorname{Re} \eta}\left[\frac{\partial^{2} w}{\partial z^{2}}+\frac{\partial^{2} w}{\partial r^{2}}+\frac{1}{r} \frac{\partial w}{\partial r}\right]=0 .
$$

Summarizing the scaling used in this formulation, $r$ and $z$ are in units of rotor radii, whereas $u, v$, and $w$ are the radial velocity, tangential velocity perturbation, and axial velocity in units of the wall speed, respectively. The variables $\theta$ and $p$ are defined by

$$
\theta=\frac{T(r, z)-T_{0}}{T_{0}}
$$

and

$$
p=\frac{P(r, z)-P_{w^{n}}}{P_{w^{n}}},
$$


where $T$ is the local temperature, $T_{0}$ is the constant ambient temperature in the centrifuge's environment, $P$ is the locai pressure, and $P_{W}$ is the constant zerothorder pressure on the rotor wall.

For the total reflux countercurrent problem, it is more convenient to use a vorticity-stream function formulation rather than the primitive variables. The main advantages of the vorticity-stream function approach are (1) $p$ is eliminated, leaving four rather than five unknown functions, and (2) the new boundary conditions are considerably simpler than the pressure boundary conditions.

Define the stream function $\psi$ and the azimuthal component of the vorticity $\omega$ by

$$
\begin{aligned}
& \frac{\partial \psi}{\partial z}=-n u, \\
& \frac{1}{r} \frac{\partial}{\partial r}(r \psi)=n w,
\end{aligned}
$$

and

$$
\omega=\frac{\partial u}{\partial z}-\frac{\partial w}{\partial r}
$$

To derive a Poisson-like equation for $\psi$, Eqs. (49) and (50) are substituted into Eq. (51).

$$
\omega=-\frac{\partial}{\partial z}\left(\frac{1}{\eta} \frac{\partial \psi}{\partial z}\right)-\frac{\partial}{\partial r}\left[\frac{1}{r n} \frac{\partial}{\partial r}(r \psi)\right] .
$$

This reduces to

$$
-\omega n=\frac{\partial^{2} \psi}{\partial z^{2}}+\frac{\partial^{2} \psi}{\partial r^{2}}+\left(\frac{1}{r}-U^{2} r\right) \frac{\partial \psi}{\partial r}-\left(\frac{1}{r^{2}}+u^{2}\right) \psi \text {. }
$$

The equation for $\omega$ is derived by differentiating Eq. (45) with respeci to $z$, differentiating Eq. (46) with respect to $r$, and subtracting. We find that

$$
\frac{\partial^{2} \omega}{\partial z^{2}}+\frac{\partial^{2} \omega}{\partial r^{2}}+\left(\frac{1}{r}-u^{2} r\right) \frac{\partial \omega}{\partial r}-\left(\frac{1}{r^{2}}+u^{2}+u^{4} r^{2}(1+B)\right) \omega=\operatorname{Re} n\left[r \frac{\partial \theta}{\partial z}-2 \frac{\partial v}{\partial z}\right]
$$




$$
-\frac{u^{a} r^{2}(1+B)}{n}\left[\left(\frac{i}{r^{2}}+u^{2}\right) \psi+\left(u^{2} r-\frac{1}{r}\right) \frac{\partial \psi}{\partial r}-\frac{\partial^{2} \psi}{\partial r^{2}}\right] \text {. }
$$

Equations (43) and (44) must be modified to eliminate u.

$$
\frac{\partial^{2} \theta}{\partial z^{2}}+\frac{\partial^{2} \theta}{\partial r^{2}}+\frac{1}{r} \frac{\partial \theta}{\partial r}=\frac{\operatorname{Re} \operatorname{Pr}(\gamma-1) U^{2} r}{\gamma} \frac{\partial \psi}{\partial z} \equiv K r \frac{\partial \psi}{\partial z}
$$

and

$$
\frac{\partial^{2} v}{\partial z^{2}}+\frac{\partial^{2} v}{\partial r^{2}}+\frac{1}{r} \frac{\partial v}{\partial r}-\frac{v}{r^{2}}=-2 \operatorname{Re} \frac{\partial \psi}{\partial z} \text {. }
$$

Also, Eqs. (45) and (46) may be differentiated by $\frac{1}{r} \frac{\partial}{\partial r} r$ and $\frac{\partial}{\partial z}$, respectiveTy, then added to obtain a Poisson equation for $p$. This Poisson equation may be solved easily after Eqs. (53)-(56) are solved, thus it will not be considered further in this report.

For the total reflux countercurrent problem, Chebyshev polynomials of the first kind are the best choice of orthogonal expansion functions. The important properties of these functions are listed in Table I. The most important feature of these polynomials is that for large $n$, the nodes are concentrated toward $|r|=1$, where the countercurrent varies most rapidly with radius. The following heuristic argument shows why the Chebyshev polynomials are so useful. To resolve the downflow next to the rotor wall and get acceptable accuracy, we need at least one complete oscillation of the highest order basis function in the downflow. Suppose that we need to resolve a feature in the downflow region that is about 0.01 rotor radii thick. The function sin $(200 \pi r)$ has one period in $0.99 \leqslant r \leqslant 1.0$, and the Chebyshev polynomial $\mathrm{T}_{33}(r)$ has one oscillation in the same interval. This means that a 17-term Fourier-Chebyshev series for the stream function will probably give accuracy comparable to a 400-term sine series. In Sec. IV we show that a 15- or 20-term Chebyshev series adequately describes the stream function for many purposes in a high speed centrifuge, and fewer terms will suffice at lower speeds. There are other orthogonal functions, such as Chebyshev polynomials of the second kind and Legendre polynomials, which are superior to trigonometric functions, but their series do not converge as rapidiy for boundary layer problems as do the Chebyshev series we have chosen. 
TABLE I

CHEBYSHEV POLYNOMIALS OF THE FIRST KIND $T_{n}(x)$

Definition

$\delta_{m n} \Delta_{m}=\int_{-1}^{+1} T_{m}(x) T_{n}(x) \frac{d x}{\sqrt{1-x^{2}}}=\left\{\begin{array}{cl}0 & m \neq n \\ \pi / 2 & m=n \neq 0 \\ \pi & m=n=0\end{array}\right.$

Recursion Relation

$T_{n+1}(x)=2 x T_{n}(x)-T_{n-1}(x)$

First Three Polynomials

$T_{0}(x)=1$

$T_{1}(x)=x$

$T_{2}(x)=2 x^{2}-1$

Fourier-Chebyshev Expansion

$$
\begin{aligned}
& f(x)=\sum_{i=0}^{\infty} a_{i} T_{i}(x) \\
& a_{i}=\frac{1}{\Delta_{i}} \int_{-1}^{+1} T_{i}(x) f(x) \frac{d x}{\sqrt{1-x^{2}}} \\
& \Delta_{i}= \begin{cases}\pi & i=0 \\
\pi / 2 & i>0\end{cases}
\end{aligned}
$$

The differential equations are defined on a two-dimensional region $0 \leqslant r \leqslant 1$ and $-L \leqslant z \leqslant L$. We want to expand the solution in the radial direction, but the normalization integral is defined on the interval $-1 \leqslant r \leqslant 1$. Thus, we choose to extend analytically the solutions to the region $-1 \leqslant r \leqslant 0$ and $-L \leqslant z \leqslant L$. The most natural way to do this extension is to consider the symmetry properties of the solutions in a plane that bisects the rotor and contains the rotation axis.

Table II summarizes the useful symmetry properties of the solution. This choice of symmetry properties makes all terms in each of the Eqs. (53)-(56) have the 
same symmetry, permitting us to extend analytically our solutions with the symmetry rules in Table II.

Each of the polynomials $T_{n}(r)$ is even or odd if $n$ is even or odd, respectively. Therefore, we assume

$$
\begin{aligned}
& \psi \simeq \sum_{i=}^{N_{\psi}} \Psi_{2 i-1}(z) T_{2 i-1}(r), \\
& \theta \simeq \sum_{i=1}^{N_{\theta}} \theta_{2 i-2}(z) T_{2 i-2}(r), \\
& \omega \simeq \sum_{i=1}^{N} \Omega \Omega_{-1}^{N}(z) T_{2 i-1}(r),
\end{aligned}
$$

SYMMETRY PROPERTIES OF THERMAL DRIVE, TOTAL REFLUX SOLUTIONS

Symmetry Symmetry Across

Function About $r=0$ The Rotor Midplane

$\theta$

even odd

$\checkmark$ odd odd

$\psi \quad$ odd even

$\omega$ odd even

and

$$
v \simeq \sum_{i=1}^{N} v_{2 i-1}(z) T_{2 i-1}(r)
$$

Because the $T_{n}(r)$ form a complete orthogonal set, Eqs. (57)-(60) would be exact if $i$ were aliowed to approach infinity in the summations. However, we must truncate the series for numerical work to keep the number of ordinary differential equations finite.

Next we derive the equations for the expansion coefficients. We show the derivatior. in some detail for the thermal diffusion equation, and merely quote the results for the other three equations. Substituting Eqs. (57) and (58) into Eq. (55), we obtain

$$
\begin{aligned}
\sum_{i=1}^{N_{\theta}} T_{2 i-2}(r) \frac{d^{2} \theta_{2 i-2}(z)}{d z^{2}} & +\sum_{i=1}^{N_{\theta}} \theta_{2 i-2}(z) \frac{d^{2} T_{2 i-2}(r)}{d r^{2}}+\frac{1}{r} \sum_{i=1}^{N_{\theta}} \theta_{2 i-2}(z) \frac{d T_{2 i-2}(r)}{d r} \\
& +K r \sum_{i=1}^{N_{\psi}} \frac{d \Psi}{2 i-1} \frac{(z)}{d z} T_{2 i-1}(r)
\end{aligned}
$$


Now multiply Eq. (61) by $T_{2 j-2}(r) / \sqrt{1-r^{2}}$ and integrate over $-1 \leqslant r \leqslant 1$. Then

$$
\begin{aligned}
\sum_{i=1}^{N_{\theta}}\left\{\frac{d^{2} \theta_{2 i-2}(z)}{d z^{2}} \int_{-1}^{+1} T_{2 j-2}(r) T_{2 i-2}(r) \frac{d r}{\sqrt{-r^{2}}}\right. \\
\quad+\theta_{2 i-2}(z)\left[\int_{-1}^{+1} \frac{d^{2} T_{2 i-2}(r)}{d r^{2}} T_{2 j-2}(r) \frac{d r}{\sqrt{1-r^{2}}}\right. \\
\left.\left.+\int_{-1}^{+1} \frac{1}{r} \frac{d T_{2 i-2}(r)}{d r} T_{2 j-2}(r) \frac{d r}{\sqrt{1-r^{2}}}\right]\right\} \\
=K \sum_{i=1}^{N_{\psi}} \frac{d \Psi 2 i-1}{d z} \int_{-1}^{+1} T_{2 j-2}(r) T_{2 i-1}(r) r \frac{d r}{\sqrt{1-r^{2}}}
\end{aligned}
$$

Define

$$
\begin{aligned}
& I_{i j} \equiv \frac{1}{\Delta_{2 j-2}} \int_{-1}^{+1} r T_{2 j-2}(r) T_{2 i-1}(r) \frac{d r}{\sqrt{1-r^{2}}}, \\
& I_{i j}^{2} \equiv \frac{1}{\Delta_{2 j-2}} \int_{-1}^{+1} \frac{1}{r} \frac{d T_{2 i-2}(r)}{d r} T_{2 j-2}(r) \frac{d r}{\sqrt{1-r^{2}}}, \\
& I_{i j}^{3} \equiv \frac{1}{\Delta_{2 j-2}} \int_{-1}^{+1} \frac{d^{2} T_{2 i-2}(r)}{d r^{2}} T_{2 j-2}(r) \frac{d r}{\sqrt{1-r^{2}}},
\end{aligned}
$$

and

$$
\mathrm{J}_{i j}^{1} \equiv \mathrm{I}_{i j}^{3}+\mathrm{cI} \mathrm{I}_{\mathbf{i j}}^{2}
$$

Note that $I_{1 j}^{2}=I_{1 j}^{3}=0$ because $T_{0}=1$. As explained later, we are interested in $j=1,2,3, \ldots, N_{\theta}-1$. The first integral in Eq. (62) is the orthogonality relation 


$$
\int_{-1}^{+1} T_{2 j-2}(r) T_{2 i-2}(r) \frac{d r}{\sqrt{1-r^{2}}}=\delta_{i j} \Delta_{2 j-2}
$$

Using Eqs. (63)-(67), we rewrite Eq. (62) in a more compact form:

$$
\frac{d^{2} \theta_{2 j-2}(z)}{d z^{2}}+J_{j j}^{1} \theta_{2 j-2}(z)=k \sum_{i=1}^{N} \frac{d \Psi}{N_{2 j-1}(z)} \frac{1 z}{d z} I_{i j}-\sum_{\substack{i=2 \\ i \neq j}}^{N_{\theta}} \theta_{2 i-2}(z) j_{i j}^{1},
$$

$j=1,2,3, \ldots, N_{\theta}-1$. Note that the integrals in Eqs. (63)-(65) are independent of problem parameters. They may be computed and stored once and for all, regardless of Re, $L$, and $A^{2}$. Three points must be considered in evaluating the integrals where numerical integration is used. First, the integrands are even about $r=0$, so it is advantageous so perform the integrations only on the interval $0 \leqslant$ $r \leqslant 1$. Second, the integrands have singularities at $r= \pm 1$, which are easily removed by the transformation $r=\sin \xi$. Third, the integrands oscillate violently for large $i$ and $j$, and special care may be needed to get good accuracy. For some integrals the truncation error of Simpson's rule is zero because of a geometrical accident. For example, $\mathrm{I}_{31}^{3}$ may be found exactly by using Simpson's rule on three integration nodes at $\xi=0, \pi / 4$, and $\pi / 2$. However, we do not expect to have such good fortune for all the integrals, and special numerical techniques may be needed to evaluate some of them accurately.

Now we apply the same procedure to Eqs. (53), (54), and (56).

$$
\frac{d^{2} \Psi_{2 j-1}(z)}{d z^{2}}+\left(j_{j j}^{2}-y^{2}\right) \Psi_{2 j-1}(z)=-\sum_{i=1}^{N_{\psi}} j_{i j}^{3} \Omega_{2 i-1}(z)-\sum_{\substack{j=1 \\ i \neq j}}^{N_{\psi}} j_{i j}^{2} \Psi_{2 i-1}(z),
$$

$j=1,2,3, \ldots, N_{\psi}-1$, where

$$
J_{i j}^{2} \equiv I_{i j}^{5}+I_{i j}^{6}-U^{2} I_{i j}^{7}-I_{i j}^{4} \text {, }
$$




$$
\begin{aligned}
& J_{i j}^{3}=\frac{1}{\Delta 2 j-1} \int_{-1}^{+1} n(r) T_{2 j-1}(r) T_{2 i-1}(r) \frac{d r}{\sqrt{1-r^{2}}}, \\
& I_{i j}^{4}=\frac{2}{\pi} \int_{-1}^{+1} \frac{1}{r^{2}} T_{2 i-1}(r) T_{2 j-1}(r) \frac{d r}{\sqrt{1-r^{2}}}, \\
& I_{i j}^{5}=\frac{2}{\pi} \int_{-1}^{+1} \frac{d^{2} T_{2 i-1}(r)}{d r^{2}} T_{2 j-1}(r) \frac{d r}{\sqrt{1-r^{2}}}, \\
& I_{i j}^{6}=\frac{2}{\pi} \int_{-1}^{+1} \frac{1}{r} \frac{d T_{2 i-1}(r)}{d r} T_{2 j-1}(r) \frac{d r}{\sqrt{1-r^{2}}},
\end{aligned}
$$

and

$$
I_{i j}^{7}=\frac{2}{\pi} \int_{-1}^{+1} r \frac{d T_{2 i-1}(r)}{d r} T_{2 j-1}(r) \frac{d r}{\sqrt{1-r^{2}}}
$$

As before, the I's are problem independent and the J's are computed only once at the start of each problem.

Equation (54) reduces to

$$
\begin{aligned}
\frac{d^{2} \Omega_{2 j-1}(z)}{d z^{2}}+\left(j_{j j}^{4}-c U^{2}\right) \Omega_{2 j-1}(z)=-\sum_{\substack{i=1 \\
i \neq j}}^{N} j_{i j}^{4} \Omega_{2 i-1}(z)+\operatorname{Re} \sum_{i=1}^{N_{\theta}} j_{i j}^{5} \frac{d \theta_{2 i-2}(z)}{d z} \\
-2 \operatorname{Re} \sum_{i=1}^{N_{y}} j_{i j}^{3} \frac{d V_{2 j-1}(z)}{d z}-u^{4}(+B) \sum_{i=1}^{N_{\Psi}} j_{i j}^{6} \Psi_{2 i-1}(z), \quad \text { (76) }
\end{aligned}
$$

$j=1,2,3, \ldots N_{\omega}-1$, where

$$
J_{i j}^{4}=I_{i j}^{5}+I_{i j}^{6}-I_{i j}^{4}-U^{2} I_{i j}^{7}-(1+B) U^{4} I_{i j}^{8},
$$




$$
\begin{aligned}
J_{i j}^{5} & =\frac{2}{\pi} \int_{-1}^{+1} r n T_{2 i-2}(r) T_{2 j-1}(r) \frac{d r}{\sqrt{1-r^{2}}} \\
j_{i j}^{6} & =\frac{2}{\pi}\left[\int_{-1}^{+1} \frac{1}{n} T_{2 i-1}(r) T_{2 j-1}(r) \frac{d r}{\sqrt{1-r^{2}}}\right. \\
& +u^{2} \int_{-1}^{+1} \frac{r^{2}}{n} T_{2 i-1}(r) T_{2 j-1}(r) \frac{d r}{\sqrt{1-r^{2}}} \\
& +U^{2} \int_{-1}^{+1} \frac{r^{3}}{n} \frac{d T_{2 i-1}(r)}{d r} T_{2 j-1}(r) \frac{d r}{\sqrt{1-r^{2}}} \\
& -\int_{-1}^{+1} \frac{r}{n} \frac{d T_{2 i-1}(r)}{d r} T_{2 j-1}(r) \frac{d r}{\sqrt{1-r^{2}}} \\
& \left.-\int_{-1}^{+1} \frac{r^{2}}{n} \frac{d^{2} T_{2 i-1}(r)}{d r^{2}} T_{2 j-1}(r) \frac{d r}{\sqrt{1-r^{2}}}\right]
\end{aligned}
$$

(78)

and

$$
I_{i j}^{8}=\frac{2}{\pi} \int_{-1}^{+1} r^{2} T_{2 i-1}(r) T_{2 j-1}(r) \frac{d r}{\sqrt{1-r^{2}}}
$$

Finally, Eq. (56) becomes

$$
\frac{d^{2} V_{2 j-1}(z)}{d z^{2}}+j_{j j}^{7} V_{2 j-1}(z)=-\sum_{\substack{i=1 \\ i \neq j}}^{N_{v}} j_{i j}^{7} V_{2 i-1}(z)-2 \operatorname{Re} \frac{d \Psi_{2 j-1}(z)}{d z},
$$

$j=1,2,3, \ldots, N_{v}-1$, where

$$
J_{i j}^{7}=I_{i j}^{5}+I_{i j}^{6}-I_{i j}^{4}
$$

16 
This completes the derivation of the ordinary differential equations. Note that we reduced four second-order linear two-dimensional partial differential equations with variable coefficients to $N_{\psi}+N_{\omega}+N_{v}+N_{\theta}-4$ sc cond-order linear ordinary differential equations with constant coefficients.

Let us consider the boundary conditions for total reflux thermal drive solutions. Because of the symmetry properties of the solution, we need to consider only half the rotor, say the upper half. Furthermore, the symmetry in the radial direction automatically enforces the proper boundary conditions at $r=-1$ if they are enforced at $r=1$.

The boundary condition on the stream function is quite important and deserves special comment. We note that the boundary of the rectangle $-1 \leqslant r \leqslant 1,-L \leqslant z \leqslant L$ and the line $r=0$ are streamlines. The stream function vanishes on the axis, which implies that $\psi$ vanishes everywhere on the boundary of the rectangle. We impose a no-slip condition on the boundaries $r= \pm 1$, so Eq. (50) implies that $\frac{\partial \psi}{\partial r}$ also vanishes on the rotor wall.

The conditions imposed on the rotor wall are

$$
\begin{aligned}
& \psi(1, z)=v(1, z)=0, \\
& \theta(1, z)=\theta_{0} z,
\end{aligned}
$$

where $\theta_{0}$ is a constant, and

$$
\omega(1, z)=-\frac{\partial^{2} \psi(1, z)}{\partial r^{2}} \text {. }
$$

Equation (85) follows easily from Eq. (53). On the endcaps,

$$
\begin{aligned}
& \theta(r, L)=\theta_{0} L, \\
& v(r, L)=\psi(r, L)=0,
\end{aligned}
$$

and

$$
\omega(r, L)=-\frac{1}{n} \frac{\partial^{2} \psi(r, L)}{\partial z^{2}} .
$$

At the midplane,

$$
v(r, 0)=\theta(r, 0)=\frac{\partial \psi(r, 0)}{\partial z}=\frac{\partial \omega(r, 0)}{\partial z}=0 .
$$


Normally, one would choose a set of expansion functions so that each function satisfies the boundary conditions on the rotor wall. Hovever, $T_{n}(1)=1$, so we must use a special procedure to insure that each series satisfies the boundary conditions. There are several ways to do this. We have chosen to satisfy boundary conditions by calculating the highest order expansion coefficient from the boundary condition rather than from its differential equation. For example, choosing $\theta_{2 N_{\theta}-2}(z)$ such that

$$
\theta_{2 N_{\theta}-2}(z)=\theta_{0} z-\sum_{i=1}^{N_{\theta}-1} \theta_{2 i-2}(z)
$$

for all $z$ insures that Eq. (84) is satisfied. Similar equations may be written for $\Omega_{2 N_{\omega}-1}(z), \Psi_{2 N_{\psi}-1}(z)$, and $v_{2 N_{v}-1}(z)$.

The boundary conditions $\theta_{2 i-2}(0), \theta_{2 i-2}\left(i_{-}\right)$, etc., are found by taking the Fourier-Chebyshev transform of the boundary conditions at the midplane and on the endcaps, making sure that the boundary conditions at $r=1$ are satisfied. Most of the boundary conditions are quite simple for the particular problem we are dis.cussing. For example,

$$
\theta_{0}(L)=\theta_{0} L
$$

and

$$
\theta_{2 i-2}(L)=0, i=2,3, \ldots, N_{\theta} .
$$

We get similar relations for the other variables.

$$
\begin{aligned}
& \Omega_{2 N-1}(z)=-\sum_{i=1}^{N} \Psi_{2 i-1}(z) \frac{d^{2} T_{2 i-1}(1)}{d r^{2}}-\sum_{i=1}^{N_{\omega}-1} \Omega_{2 i-1}(z), \\
& \Omega_{2 j-1}(L)=-\sum_{i=1}^{N_{\psi}} \frac{d^{2} \psi_{2 i-1}(L)}{d z^{2}} j_{i j}^{7}, \\
& j=1,2,3,4, \ldots, N_{\omega}-1,
\end{aligned}
$$




$$
\begin{aligned}
& J_{i j}^{7}=\frac{2}{\pi} \int_{-1}^{+1} \frac{1}{n} T_{2 i-1}(r) T_{2 j-1}(r) \frac{d r}{\sqrt{1-r^{2}}}, \\
& v_{2 N_{v}-1}(z)=-\sum_{i=1}^{N_{v}-1} v_{2 i-1}(z),
\end{aligned}
$$

and

$$
\Psi_{2 N_{\psi}-1}(z)=-\sum_{i=1}^{N_{\psi}-1} \Psi_{2 i-1}(z) .
$$

All of the expansion coefficients are zero for variables that are zero at the midplane or endcap.

We have finished deriving the set of ordinary differential equations and their boundary conditions. The equations are a set of coupled undamped harmonic oscillator equations with mixed boundary conditions. We have shown how to make the series obey the boundary conditions on the rotor wall, al though the individual expansion functions do not obey these boundary conditions.

\section{NUMERICAL METHOD}

We wish to solve the set of second-order ordinary differential equations $(68),(69),(76)$, and (81). We recommend solving them numerically as a practical matter. It might be possible to do a normal mode or Fourier analysis, however, the amount of algebra involved would be tremendous. One might expand the equations in a Fourier-Chebyshev series axially and obtain a very large set of coupled linear algebraic equations. However, if we resolve the Ekman layer, many more terms will be needed in the axial expansion than in the radial expansion. However, if we apply the Carrier-Maslen Ekman layer theory ${ }^{1}$ at the endcaps rather than a no-slip condition, the two-dimensional expansion might be feasible. Numerical solutions on a nonuniform mesh appear to be the easiest way to get solutions. Because the two boundary conditions for each equation are given at opposite ends of the mesh, a shooting method would have to be used if one wishes to use the standard predictor-corrector or Runge-Kutta schemes. The large number of equations makes this procedure impractical. Either integral equation methods or difference schemes appear to be suitable. We have chosen the second approach and present a set of difference equations below that may be solved quite easily. 
Consider the mesh shown in Fig. 1 , which has $M$ real cells plus one fictitious cell at each end. We specify the cell edge positions $z_{k-\frac{1}{2}}, k=1,2, \ldots$, $M+3$, at the start of a problem. Generally, the cell widths will differ. We need small cells near $z=L$ to resolve the Ekman layer, but much larger cells will give us adequate resolution near $z=0$. There are two limitations on cell sizes that must te observed for accuracy and simplicity in programming. First, the three cells on each end of the mesh should be the same size, but the cell size $z_{3 / 2}{ }^{-z_{1 / 2}}$ can be different than $z_{M+5 / 2}-z_{M+3 / 2}$. This requirement greatly simplifies the treatment of boundary conditions. Second, to obtain accurate solutions, we must impose the approximate restriction

$$
\frac{2}{3} \lessgtr \frac{z_{k+\frac{1}{2}}-z_{k-\frac{1}{2}}}{z_{k+3 / 2}-z_{k+\frac{1}{2}}}=\frac{1}{f} \lessgtr 1.5
$$

If two adjacent cells are too different in width, large truncation errors are introduced into the second derivatives with respect to $z$. Experience has shown that if Eq. (98) is violated, the truncation errors due to the nonuniform mesh can become comparable to the truncation errors due to differencing on a uniform mesh. The heuristic truncation error analysis described by $\mathrm{Hirt}^{2}$ inay be used to show this effect. Let $f$ be the ratio of the widths of two adjacent cells as defined in Eq. (98). Then our difference scheme yields, for some function $y$,

$$
\begin{aligned}
\frac{\partial^{2} y}{\partial z^{2}} & \simeq \frac{2}{(1+f) \delta z}\left[\frac{y_{k+3 / 2}-y_{k+\frac{1}{z}}}{f \delta z}-\frac{y_{k+\frac{1}{2}}-y_{k-\frac{1}{z}}}{\delta z}\right] \simeq \frac{\partial^{2} y}{\partial z^{2}}+\frac{(f-1) \delta z}{3} \frac{\partial^{3} y}{\partial z^{3}} \\
& +\frac{\left(f^{2}-f+1\right) \delta z^{2}}{2} \frac{\partial^{4} y}{\partial z^{4}}, \quad \text { (99) }
\end{aligned}
$$

where $y_{k+\frac{1}{2}}=y\left(z_{k+z_{2}}\right)$ and $\delta z=z_{k+z_{2}}-z_{k-\frac{1}{2}}$. We see that our difference scheme is second-order accurate on a uniform mesh $(f=1)$, but only first-order accurate on a nonuniform mesh.

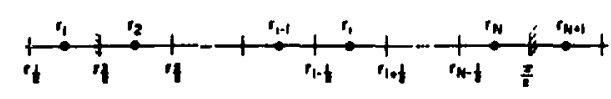

Fig. 1.

The mesh in the axial direction. The rotor midplane is at $z_{3 / 2}$, and the upper endcap is at $\mathrm{z}_{\mathrm{M}+3 / 2^{\circ}}$. 
In addition to cell edge positions $z_{k-\frac{1}{2}}$, we will also need cell center positions

$$
z_{k}=\frac{1}{2}\left(z_{k+\frac{1}{2}}+z_{k-\frac{1}{2}}\right)
$$

and the average positions of cell centers

$$
\bar{z}_{k+\frac{1}{2}}=\frac{1}{2}\left(z_{k}+z_{k+1}\right)
$$

We assume that $\Omega_{2 j-1}(z)$ and $\Psi_{2 j-1}(z)$ are defined on cell edges, whereas $v_{2 j-1}(z)$ and $\theta_{2 j-2}(z)$ are defined at cell centers. Let a superscript indicate the value of $z$ at. which a dependent variable is defined. Then the difference equations for all interior points are

$$
\begin{aligned}
\bar{u}_{k}^{1}\left(\theta_{2 j-2}^{k+1}-\right. & \left.\theta_{2 j-2}^{k}\right)-D_{k}^{2}\left(\theta_{2 j-2}^{k}-\theta_{2 j-2}^{k-1}\right)+J_{j j}^{1} \theta_{2 j-2}^{k}=k \sum_{i=1}^{N_{\psi}} I_{i j}^{1} \frac{\Psi_{2 i-1}^{k+\frac{1}{2}}-\Psi_{2 i-1}^{k-\frac{1}{2}}}{z_{k+\frac{k}{2}}-z_{k-\frac{1}{2}}} \\
& -\sum_{\substack{i=2 \\
i \neq j}}^{N_{\theta}} j_{i j}^{1} \theta_{2 i-2}^{k},
\end{aligned}
$$

$k=2,3, \ldots, M+1$ and $j=1,2,3, \ldots, N_{\theta}-1$,

$$
\begin{gathered}
D_{k-\frac{1}{2}}^{3}\left(\Psi_{2 j-1}^{k+\frac{1}{2}}-\Psi_{2 j-1}^{k-\frac{1}{2}}\right)-D_{k-\frac{1}{2}}^{4}\left(\Psi_{2 j-1}^{k-\frac{1}{2}}-\Psi_{2 j-1}^{k-3 / 2}\right)+\left(j_{j j}^{2}-u^{2}\right) \Psi_{2 j-1}^{k-\frac{1}{2}} \\
=-\sum_{i=1}^{N} j_{i j}^{3} \Omega_{2 i-1}^{k-\frac{1}{2}}-\sum_{\substack{i=1 \\
i \neq j}}^{N} j_{i j}^{2} \Psi_{2 i-1}^{k-\frac{1}{2}},
\end{gathered}
$$

$k=2,3, \ldots, M+1$ and $j=1,2,3 \ldots, N_{\psi}-1$, 


$$
\begin{aligned}
D_{k-\frac{1}{2}}^{3}\left(\Omega_{2 j-1}^{k+\frac{1}{2}}-\Omega_{2 j-1}^{k-\frac{1}{2}}\right)-D_{k-\frac{1}{2}}^{4}\left(\Omega_{2 j-1}^{k-\frac{1}{2}}-\Omega_{2 j-1}^{k-3 / 2}\right)+\left(j_{j j}^{4}-U^{2}\right) \Omega_{2 j-1}^{k-\frac{1}{2}} \\
=-\sum_{\substack{i=1 \\
i \neq j}}^{N_{\omega}} j_{i j}^{4} \Omega_{2 i-1}^{k-\frac{1}{2}}+\operatorname{Re} \sum_{i=1}^{N_{\theta}} j_{i j}^{5} \frac{\theta_{2 i-2}^{k}-\theta_{2 i-2}^{k-1}}{z_{k}-z_{k-1}} \\
-2 \operatorname{Re} \sum_{j=1}^{N_{v}} J_{i j}^{3} \frac{v_{2 i-1}^{k}-v_{2 i-1}^{k-1}}{z_{k}-z_{k-1}}-U^{4}(1+B) \sum_{j=1}^{N_{\psi}} j_{i j}^{6} \Psi_{2 i-1}^{k-\frac{1}{z}},
\end{aligned}
$$

$k=2,3, \ldots, M+1$ and $j=1,2,3, \ldots, N_{\omega}-1$, and

$$
\begin{gathered}
D_{k}\left(v_{2 j-1}^{k+1}-v_{2 j-1}^{k}\right)-D_{k}^{2}\left(v_{2 j-1}^{k}-v_{2 j-1}^{k-1}\right)+J_{j j}^{7} v_{2 j-1}^{k}=-\sum_{\substack{j=1 \\
i \neq j}}^{N_{y}} j_{i j}^{7} v_{2 i-1}^{k} \\
-2 \operatorname{Re} \frac{\Psi_{2 j-1}^{k+\frac{1}{2}}-\Psi_{2 j-1}^{k-\frac{1}{2}}}{z_{k+\frac{1}{2}}-z_{k-\frac{1}{2}}},
\end{gathered}
$$

$k=2,3, \ldots, M+1$ and $j=1,2,3, \ldots, N_{v}-1$, where

$$
\begin{aligned}
& D_{k}^{1}=\left[\left(z_{k+1}-z_{k}\right)\left(\bar{z}_{k+\frac{1}{2}}-\bar{z}_{k-\frac{1}{2}}\right)\right]^{-1,}, \\
& D_{k}^{2}=\left[\left(z_{k}-z_{k-1}\right)\left(\bar{z}_{k+\frac{1}{2}}-\bar{z}_{k-\frac{1}{2}}\right)\right]^{-1,}, \\
& D_{k-\frac{1}{2}}^{3}=\left[\left(z_{k+\frac{1}{2}}-z_{k-\frac{1}{2}}\right)\left(z_{k}-z_{k-1}\right)\right]^{-1,},
\end{aligned}
$$

and

$$
D_{k-\frac{1}{2}}^{4}=\left[\left(z_{k-\frac{1}{2}}-z_{k-3 / 2}\right)\left(z_{k}-z_{k-1}\right)\right]^{-1} \text {. }
$$


Boundary conditions are used to close the set of Eqs. (102)-(105). If we treat the endcap as a rigid wall in the traditional way, then we would impose a boundary condition such as (91) in the following way:

$$
\frac{1}{2}\left(\theta_{0}^{M+2}+\theta_{0}^{M+1}\right)=\theta_{0} L
$$

However, Eq. (110) introduces a large zeroth-order truncation error for $\frac{d^{2} \theta_{0}}{d z^{2}}$, as noted by Buzbee. ${ }^{3}$ The zeroth-order error may be eliminated by using

$$
\left(\frac{d^{2} \theta_{0}}{d z^{2}}\right)^{K=M+3 / 2} \simeq\left[\frac{\theta_{0} L-\theta_{0}^{M+1}}{0.5 \delta z}-\frac{\theta_{0} L-\theta_{0}^{M}}{1.5 \delta z}\right] \frac{1}{\delta z}=\frac{1}{\delta z^{2}}\left[\theta_{0}^{M+2}-2 \theta_{0}^{M+1}+\theta_{0}^{M}\right] .
$$

Solving for $\theta_{0}^{M+2}$,

$$
\theta_{0}^{M+2}=\frac{8}{3} \theta_{0} L+\frac{1}{3} \theta_{0}^{M}-2 \theta_{0}^{M+1}
$$

This boundary condition assumes that the first three cells at the end of the mesh have the same $\delta z$. Similarly, we obtain

$$
\theta_{2 j-2}^{M+2}=\frac{1}{3} \theta_{2 j-2}^{M}-2 \theta_{2 j-2}^{M+1}, \quad j=2,3, \ldots, N_{\theta}-1,
$$

$\operatorname{arrd}$

$$
\theta_{2 j-2}^{1}=\frac{1}{3} \Theta_{2 j-2}^{3}-2 \theta_{2 j-2}^{2}, j=1,2, \ldots, N_{\theta}-1 .
$$

The highest order coefficients are evaluated from Eq. (90). Note that even though $\theta_{2 j-2}$ is antisymmetric about $z=0$, we use an equation such as Eq. (111) rather than antisymmetry to define $\Theta_{2 j-2}^{1}$ to eliminate the zeroth-order error in $\frac{d^{2} \theta_{2 j-1}^{2}}{d z^{2}}$. For $v_{2 j-1}$, 


$$
v_{2 j-1}^{M+2}=\frac{1}{3} v_{2 j-1}^{M}-2 v_{2 j-1}^{M+1}
$$

and

$$
v_{2 j-1}^{1}=\frac{1}{3} v_{2 j-1}-2 v_{2 j-1}^{2}
$$

for $j=1,2, \ldots, N_{v}-1$. Use Eq. (96) to compute $v_{2 N_{v}-1}$.

Boundary conditions for $\Psi_{2 j-1}$ and $\Omega_{2 j-1}$ are a little different because these variables are defined on cell edges rather than cell centers. Both are even about $z=0$, so we take

$$
\Psi_{2 j-1}^{1 / 2}=\Psi_{2 j-1}^{5 / 2}
$$

and

$$
\Omega_{2 j-1}^{1 / 2}=\Omega_{2 j-1}^{5 / 2}
$$

for all $j$. The situation at the endcap is considerably more complex than it is at the midplane. Because $\psi(r, L)=0$, we have

$$
\psi_{2 j-1}^{M+3 / 2}=0
$$

for all j. We do not need $\psi_{2 j-1}^{M+5 / 2}$ in Eq. (103), but we do need it for Eq. (94). We obtain $\psi_{2 j-1}^{\mathrm{M}+5 / 2}$ by assuming $u$ is defined at cell centers, differencing Eq. (49), and then setting $u=0$ on the endcap. The result is

$$
\Psi_{2 j-1}^{M+5 / 2}=\frac{7}{3} \psi_{2 j-1}^{M+1 / 2}-\frac{1}{3} \psi_{2 j-1}^{M-1 / 2}
$$

$j=1,2, \ldots, N_{\psi}-1$. Use Eq. (97) to compute $\Psi_{2 N_{\psi}-1}^{M+5 / 2}$. 
From Eq. (94),

$$
\Omega_{2 j-1}^{M+3 / 2}=-\sum_{i=1}^{N} \frac{J_{i j j}^{7}}{\delta z^{2}}\left(\psi_{2 j-1}^{M+5 / 2}+\psi_{2 j-1}^{M+1 / 2}\right),
$$

$j=1,2,3, \ldots, N_{\omega}-1$. From Eq. (93),

$$
\Omega_{2 N}^{M+3 / 2}=-\sum_{i=1}^{N_{\omega}-1} \Omega_{2 i-1}^{M+3 / 2}
$$

because $\omega=0$ in the corner $r=1$ and $z=L$. We do not need $\Omega_{2 j-1}^{M+5 / 2}$.

With ail these boundary conditions, we have as many iinear equations as unknowns, and in principle we can solve this system. We have not attempted to solye this problem, nor have we examined the radial integrais to see if any of them vanish, thereby simplifying the problem. Although direct solution is probably not feasible for any reasonable set of integration nodes and number of terms in the series, there is no obvious rason why we expect any difficulty in finding an iterative scheme that converges and is, nopefully, fairly efficient.

\section{SIMPLE NUMERICAL EXAMPLE AND MISCELLANEOUS CONSIDERATIONS}

Here we present a numerical solution of a diffusion equation using the technique discussed in the previous sections, and we estimate the number of terms needed in the various series for the centrifuge problem. We also show some of the convergence properties of the series and how the equations may simplify if some of the radial integrals vanish. Application of the technique to the isotope diffusion equation is briefiy discussed.

Consider the following problem:

$$
\frac{\partial^{2} \theta}{\partial x^{2}}+\frac{\partial^{2} \theta}{\partial y^{2}}+\frac{\pi^{2}}{2} \theta=0
$$

on the rectangle $-1 \leqslant x \leqslant+1,0 \leqslant z \leqslant 1$, with the boundary conditions

$$
\theta( \pm 1, y)=\theta(x, 1)=0
$$


and

$$
\theta(x, 0)=\cos (\pi x / 2)
$$

The analytic solution to this problem is

$$
\theta(x, y)=\cos (\pi x / 2) \cos (\pi y / 2)
$$

Assume

$$
\theta(x, y)=\sum_{i=1}^{N} \theta_{2 i-2}(y) T_{2 i-2}(x),
$$

where

$$
\Theta_{2 i-1}(y)=\frac{1}{\Delta_{2 i-2}} \int_{-1}^{+1} T_{2 i-2}(x) \theta(x, y) \frac{d x}{\sqrt{1-x^{2}}}
$$

The boundary conditions on the expansion coefficients are

$$
\begin{aligned}
& \theta_{2 i-2}(1)=0, \\
& \theta_{0}(y)=-\sum_{i=2}^{N} \theta_{2 i-2}(y),
\end{aligned}
$$

and

$$
\theta_{2 i-1}(0)=\frac{2}{\pi} \int_{-1}^{+1} T_{2 i-2}(x) \cos (\pi x / 2) \frac{d x}{\sqrt{1-x^{2}}}
$$

for $i=2,3, \ldots, N$. Note that we are imposing the boundary condition at $x=1$ with the proper choice of $\theta_{0}$ rather than $\theta_{2 \mathrm{~N}-2}$. We discuss the reason for this choice later in this section. 
The ordinary differential equations are

$$
\frac{d^{2} \theta_{2 j-2}(y)}{d y^{2}}+\left(I_{j j}+\frac{\pi}{2}^{2}\right) \theta_{2 j-2}(y)=-\sum_{\substack{i=1 \\ i \neq j}}^{N} I_{i j} \theta_{2 i-2}(y),
$$

where

$$
I_{i j}=\frac{1}{\Delta_{2 j-2}} \int_{-1}^{+1} \frac{d^{2} T_{2 i-2}(x)}{d x^{2}} T_{2 j-2}(x) \frac{d x}{\sqrt{1-x^{2}}} .
$$

Clearly, $I_{1 j}=0$. We found that $I_{i j}$ also vanishes if $j \geqslant i$. Table III shows $a$ few values of $I_{1 j}$. Equation (132) then becomes

$$
\frac{d^{2} \theta_{2 j-2}(y)}{d y^{2}}+\frac{\pi^{2}}{2} \theta_{2 j-2}(y)=\left\{\begin{array}{l}
-\sum_{i=j+1}^{N} I_{i j} \theta_{\left.2 i-2^{i y}\right)} \text { for } j \neq N \\
0 \text { for } j=N
\end{array} .\right.
$$

Thus, in this case the problem is greatly simplified because for a fixed $j$, $\theta_{2 j-2}(y)$ is independent of the $\theta_{2 i-2}(y), i<j$. In particular, $\theta_{2 N-2}(y)$ does not depend on any other expansion coefficients, $\theta_{2(N-1)-2}(y)$ depends only on $\theta_{2 N-2}(y)$, etc. This means that no iterative procedure is needed. We merely start solving Eq. (134) with $j=N$, then solve for the next lower order coefficient until we reach $j=2$. Then the $j=1$ coefficient is found from Eq. (130). The vanishing coupling integrals, plus our choice of boundary conditions, greatly simplify this problem. If we had chosen to enforce che boundary condition using $\theta_{2 \mathrm{~N}-2}(y)$, then an iterative procedure would have been required.

The difference approximation to Eq. (134) is

$$
\frac{1}{\delta y^{2}} \theta_{2 j-2}^{k-1}+\left(\frac{\pi^{2}}{2}-\frac{2}{\delta y^{2}}\right) \theta_{2 j-2}^{k}+\frac{1}{\delta y^{2}} \theta_{2 j-2}^{k+1}=\left\{\begin{array}{l}
-\sum_{i=j+1}^{N} I_{i j} \theta_{2 i-2}^{k} \text { for } j=2,3 \ldots, \\
\text { for } j=2,3, \ldots, N-1 \\
0 \text { for } j=N .
\end{array}\right.
$$


TABLE III

YALUES OF THE RADIAL INTEGRAL $I_{i j}$

$\begin{array}{rrr}i & \underline{j} & \mathbf{I}_{\mathbf{i j}} \\ 2 & 1 & 4 \\ 3 & 1 & 32 \\ 3 & 2 & 48 \\ 4 & 1 & 108 \\ 4 & 2 & 192 \\ 4 & 3 & 120 \\ 5 & 1 & 256 \\ 5 & 2 & 480 \\ 5 & 3 & 384 \\ 5 & 4 & 224\end{array}$

We solved these difference equations on a rather coarse ten-step mesh,

$$
z_{k}=0.1(k-1), k=1,2, \ldots, 11,
$$

using a direct tridiagonal linear equation solver. Figure 2 shows the solution as a function of $x$ for $y=0.6$, and Fig. 3 shows the solution as a function of $y$ for $x=0.6$. These results are typical in their accuracy and convergence properties. Numerical data are plotted for $N=3,5,7$, and 9 . For $N=9$, the numerical solution has converged to the point that almost no difference is seen on the graphs between it and the

analytic solution. The solution for $\mathrm{N}=3$ is not very good. However, one can see that the numerical errors are reduced by approximately a factor of 3 every time two more ierms are added to the series. This behavior illustrates the fact that when the Chebyshev series begins to converge, it converges with only a small number of additional terms.

Great care is needed in choosing the procedure for enforcing the boundary conditions at $r=1$. In our simple numerical example, we were fortunate that the nature of the coupling let us impose boundary conditions by varying the lowest order coefficient. This procedure generally will not work because we lose the information contained in the lowest order differential equation. We succeeded in our example because the lowest order mode does not appear in the differential equation for any other mode. Generally, we cannot prove convergence of the series expansions when we impose boundary conditions using the lowest order mode. However, convergence can be proved if we vary the highest order mode to satisfy boundary conditions.

This problem has also been solved using the highest order coefficient to satisfy the boundary conditions. For a given error, this procedure was found to require only about half as many terms in the series. 
The diffusion problem is well behaved because the solution is very smooth. We cannot expect the centrifuge problefi to be solved with only a few terms in each series, nor do we expect to need an unmanageable number of terms. To estimate the number of terms needed for a high speed centrifuge, we took the Fourier-Chebyshev transform of the stream function for $A^{2}=25$, which is the highest speed considered by Parker and Mayo. ${ }^{4}$ The numerical results are shown in Fig. 4. The curve is the analytic stream function, and the numerical data are for series with 5, 10,15, and 20 terms. Note that 20 terms are required for good convergence, although a 15-term series may be adequate for some purposes. If we assume that 20 terms per series are adequate, then we will be solving 80 ordinary differential equations. Even if a few more terms are needed, present computers can handle the problem. A nice feature of the method is that if, for example, 15 terms turn out to be insufficient, it is simple to compute a 20 -term solution because the 15-term solution provides a good initial condition for the new calculation. Slower centrifuges can be modeled with fewer terms in each series.

Once we have a hydrodynamics solution in the form of expansion coefficients of the Chebyshev series, it is natural to solve the isotope diffusion equation using the finite Fourier-Chebyshev transform. Assume the concentration of the desired isotope $\xi(r, z)$, is expanded as

$$
\xi(r, z)=\sum_{i=1}^{N_{\xi}} x_{2 i-2}(z) T_{2 i-2}(r) .
$$

We now wish to reduce the diffusion equation to a set of ordinary differential equations. The procedure is completely analogous to the two problems examined earlier in this report, so we will not include the equations. However, note that the nonlinear term [Eq. (138)] may be treated by our transform method.

$$
\begin{aligned}
\frac{1}{r} \frac{\partial}{\partial r}\left(r^{2} \xi^{2}\right) & =\frac{1}{r} \frac{\partial}{\partial r}\left[r^{2} \sum_{i=1}^{N_{\xi}} x_{2 i-2}(z) T_{2 i-2}(r) \sum_{j=1}^{N_{\xi}} x_{2 j-2}(z) T_{2 j-2}(r)\right] \\
& =\sum_{i=1}^{N_{\xi}} \sum_{j=1}^{N_{\xi}} x_{2 i-2}(z) x_{2 j-2}(z) \frac{1}{r} \frac{\partial}{\partial r}\left[r^{2} T_{2 i-2}(r) T_{2 j-2}(r)\right] .
\end{aligned}
$$




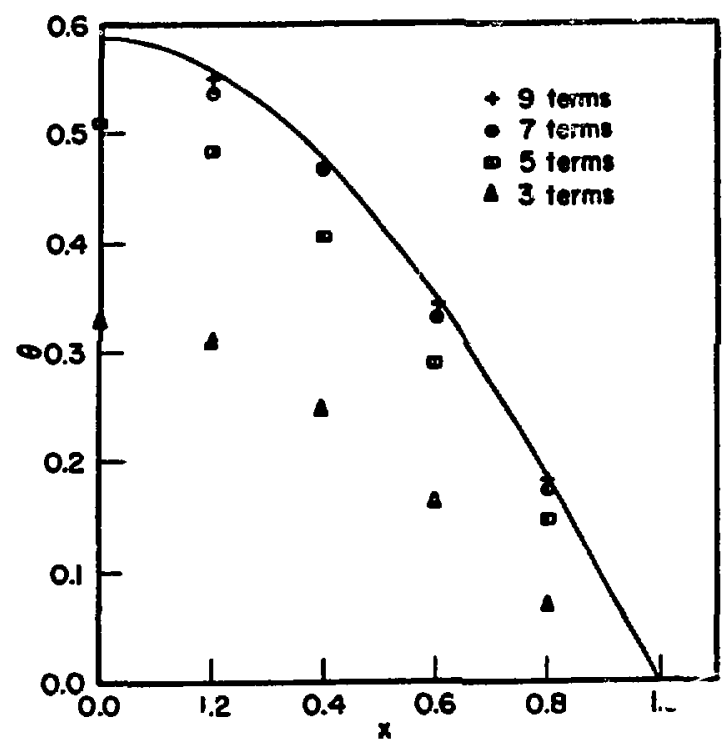

Fig. 2.

Solution to the siniple diffusion problem. The solid curve is the analytic solution for $y=0.6$, and the single points are numerical solutions with different numbers of terms in the series expansion.

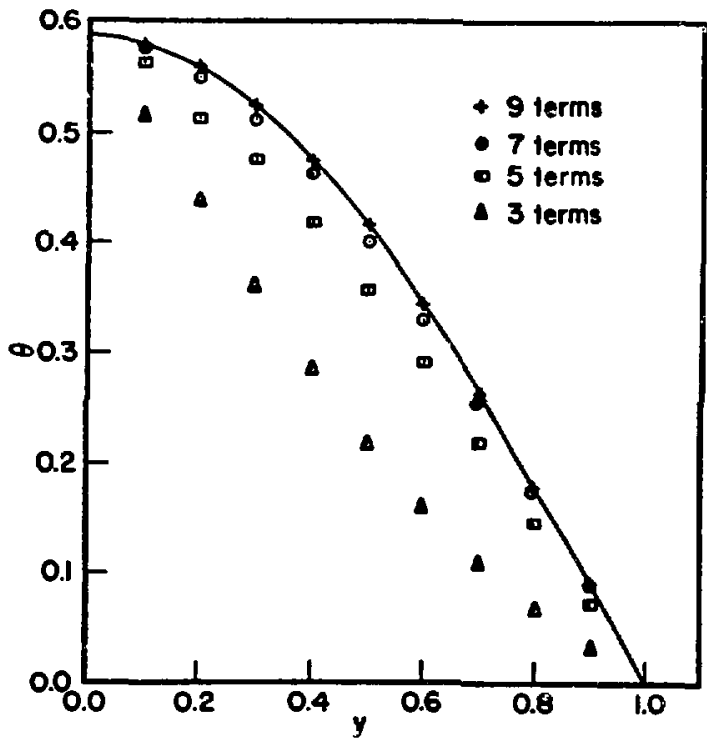

Fig. 3.

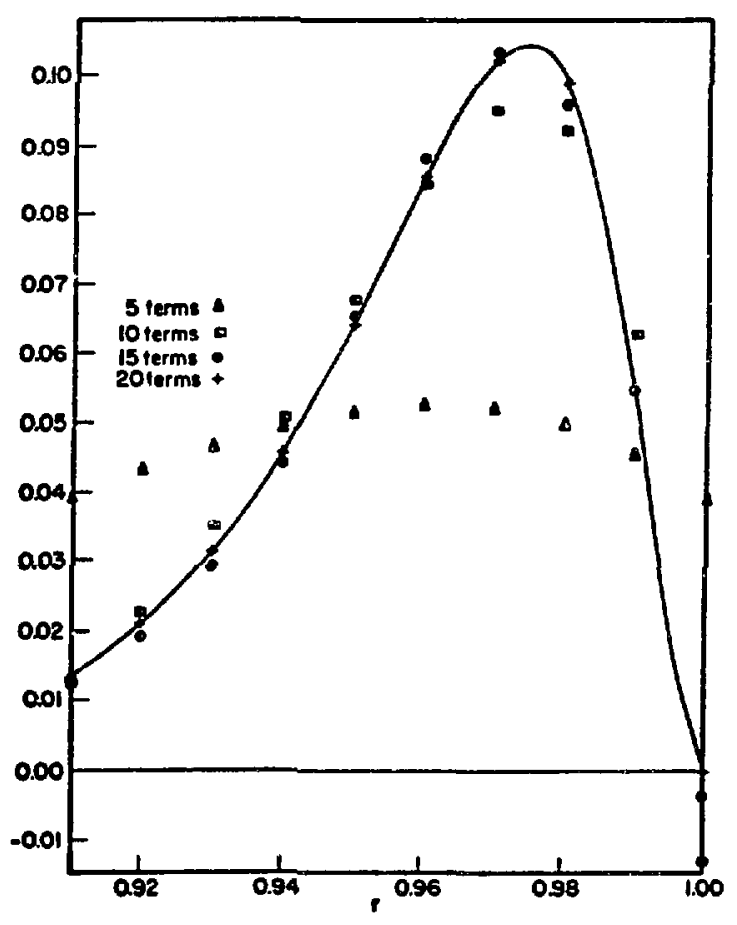

Fig. 4.
Solution to the simple diffusion problem. The solid curve is the analytic solution for $x=0.6$, and the single points are numerical solutions with different numbers of terms in the series expansion.
The solid curve is the stream function for the Universal High Speed Profile with $A^{2}=25$. The plotted points represent four different Chebyshev series with different numbers of terms. 
The ordinary differential equation for $x_{2 \ell-2}(z)$ will then contain a term proportional to

$$
\sum_{i=1}^{N_{\xi}} \sum_{j=1}^{N_{\xi}} x_{2 i-2}(z) x_{2 j-2} \int_{-1}^{+1} T_{22-2}(r) \frac{1}{r} \frac{\partial}{\partial r}\left[r^{2} T_{2 i-2}(r) T_{2 j-2}(r)\right] \frac{d r}{\sqrt{1-r^{2}}} .
$$

In this particular case, the nonlinear term is easy to include and should riut slow down the computer program unacceptably. However, care must be used in doing multiple sums on a computer because the number of arithmetic operations can be yery large for an apparently innocuous sum. For Eq. (139), we need 400 adds and 800 multiplies to do the sum once if $N_{\xi}=20$. We hopt that some of the radial integrals vanish, reducing the required number of operations. Fortunately, the nonlinear term is not important for many applications where $\xi<<1$.

The finite Fourier-Chebyshev transform method may also be applied to problems with feed and scoops. The appropriate volume source terms for mass, momentum, and energy are added to each of the five partial differential equations (42)(46). The volume source terms model azimuthally averaged shock effects and mass withdrawa 1 and introduction.

The presence of a mass volume source term forces us to redefine the stream function. Equations (49) and (50) do not give us mass conservation if

$$
\frac{1}{r} \frac{\partial}{\partial r}(r n u)+\frac{\partial(\eta w)}{\partial z}=Q \neq 0 .
$$

We introduce the mass flux potential $\phi$, which allows us to satisfy Eq. (140). Let

$$
\frac{\partial \phi}{\partial z}+\frac{1}{r} \frac{\partial}{\partial r}(r \psi)=\eta w
$$

and

$$
-\frac{\partial \phi}{\partial r}+\frac{\partial \psi}{\partial z}=-n u \text {. }
$$


Substituting Eqs. (141) and (142) into Eq. (140), we find that

$$
\frac{1}{r} \frac{\partial}{\partial r}\left(r \frac{\partial \phi}{\partial r}\right)+\frac{\partial^{2} \phi}{\partial z^{2}}=Q
$$

The function $\phi$ vanishes on all machine walls, $\phi$ is even about $r=0$, the expansion coefficients of $\phi$ are cell centered, and the symmetry of $\phi$ about the rotor midplane is opposite that of $\psi$. Note that the boundary conditions on $\psi$ now depend on $\phi$, and the physical interpretation of $\psi$ is not the same as it is for problems with $Q=0$. Equation (143) is very similar to our numerical example, and should be solved just as easily once the Fourier-Chebyshev transform of $Q$ is calculated. If we assume the source terms are dependent only on the zeroth-order flow field, then Eq. (143) is solved only once at the beginning of a problem. If we couple $Q$ to the perturbations, then Eq. (43) must be included in the iteration loop. Either approach should add little extra difficulty to finding solutions. Nonlinear terms may be included in the hydrodynamical calculation in the same way as the $\xi^{2}$ term in the isotope diffusion equation. Because the transformations of Eq. (143) and Eqs. (42)-(46) with source terms and/or nonlinear terms are straighforward, we will not discuss them further in this report.

In this discussion we have sacrificed strict mathematical rigor for the sake of clarity. Perhaps our worst sin of omission is the question of convergence of the series expansions. It can be shown both theoretically and enpirically that the Chebyshev series have excellent convergence properties. The reader interes: ed in these spectral techniques should read the paper by orszag. ${ }^{5}$

\section{ACKNOWLEDGMENTS}

I wish to express my sincere gratitude to Raymond C. Mjolsness for his help in working out several troublesome aspects of this problem. 


\section{REFERENCES}

1. G. F. Carrier and S. H. Maslen, "Flow Phenomena in Rapidly Rotating Systems," USAEC Report TID-18065 (1962).

2. C. W. Hirt, "Heuristic Stability Theory for Finite-Difference Equations," J. Comp. Phys. 2, 339 (1968).

3. B. L. Buzbee, Los Alamos Scientific Laboratory, private communication, 1973 .

4. H. M. Parker and T. T. Mayo, IV, "Countercurrent Flow in a Semi-Infinite Gas Centrifuge," Research Laboratories for the Engineering Sciences, University of Virginia, Charlottesville, VA, report number EP. $4422-279-63 \mathrm{~V}$, January 1963.

5. S. A. Orszag, "Numerical Sinulations of Incompressible Flows Within simple Boundaries. 1. Galerkin (Spectral) Rer.resentations," Studies in App. Math. $1,293(1971)$. 